\title{
Behavioral characteristics of a chondrostean sturgeon species Acipenser baerii prelarvae in response to different environmental light intensities in a diel photoperiodic cycle
}

\author{
Eun Jeong Kim ${ }^{1}$, Chulhong Park ${ }^{2}$ and Yoon Kwon Nam ${ }^{1, *}$ \\ ${ }^{1}$ Department of Marine Bio-Materials and Aquaculture, Pukyong National University, Busan 48513, Korea \\ ${ }^{2}$ Dinoville Sturgeon Aquafarm, Hamyang 50027, Korea
}

Received August 11, 2020

Revised September 17, 2020

Accepted September 17, 2020

\section{*Correspondence \\ Yoon Kwon Nam \\ E-mail: yoonknam@pknu.ac.kr}

ORCID

https://orcid.org/0000-0001-8870-2098
ABSTRACT Behavioral response to a diel photoperiodicity (500 Ix for $16 \mathrm{~h}, 5 \mathrm{~lx}$ for $4 \mathrm{~h}$ and $<0.5 \mathrm{Ix}$ for $4 \mathrm{~h}$ ) and phototactic characteristics in dark conditions were examined with Siberian sturgeon Acipenser baerii (Chondrostei, Actinopterygii) prelarvae. Siberian sturgeon prelarvae represented both qualitative and quantitative changes in their behavioral patterns according to different light intensities in a diel photoperiodicity. Under daylight conditions (500 lx), prelarvae displayed saltatory changes of behavioral features with ages (Day 0-Day 9) in a general order of swimming-up/drifting, swimming in the upper water column, benthic swimming with rheotaxis, schooling and postschooling behavior. Compared to daylight conditions, prelarvae tended to show more benthic performances and quantitative reductions of schooling and postschooling behaviors under dimlight conditions (5 Ix). Under dark conditions (< $0.5 \mathrm{Ix})$, prelarvae exhibited a fairly uniform behavioral pattern characterized by the benthic swimming across the bottom of the tank. From phototaxis tests under dark conditions, navigational responses of prelarvae to a spotlight illumination were quantitatively changed as their ages increased. The phototactic responses reached the peak on Day 2, continued until Day 4, and then gradually decreased until Day 8. A partial recovery of positive phototaxis was observed on Day 9. Data from this study suggest that the diel light cycle as well as the light intensity of each interval in the cycle should be considered as important components of a practical guide for evaluating fitness and developmental states of artificially propagated Siberian sturgeon prelarvae.

Keywords: diel photoperiodicity, light intensity, ontogenetic behavior, phototaxis in dark conditions, Siberian sturgeon Acipenser baerii

\section{INTRODUCTION}

Sturgeons belonging to Acipenseriformes represent the ancient lineage of actinopterygian fishes (ray-finned fishes), often referred to 'living fossils' (Birstein et al.,
1997; Web and Doroshov, 2011). Due to their primitive phylogenetic position, sturgeons have been considered as useful models for various evolutionary researches (Kim et al., 2005; Cho et al., 2007; Kim et al., 2019a). In addition, sturgeons, as the source of caviar, have been valuable 
fisheries resources. However, most natural populations of all acipenseriform species have been significantly endangered or threatened during last decades. For this reason, aquaculture-based propagations of sturgeons are important not only for their conservation programs but also the sustainable production of commercial caviars (Doukakis et al., 2012).

Light is one of the most critical factors to influence behavioral and physiological characteristics of sturgeons particularly during their early life stages (Rodriguez and Gisbert, 2002; Mueller and Neuhauss, 2010). Phototactic properties of sturgeon species have been known to be innately acquired at the stage of hatching, and newly hatched prelarvae (i.e., yolk sac-bearing larvae) represent a wide spectrum of behavioral interactions with environmental lights (Dettlaff et al., 1993; Kim et al., 2019b). Species-specific characteristics of photobehaviors during prelarval stages are closely related with dispersal and migration styles of the sturgeon species in natural habitats, which is undoubtedly of importance for designing restocking and conservation programs of endangered populations (Dettlaff et al., 1993; Richmond and Kynard, 1995; Gisbert et al., 1999a). With a hatchery perspective, lightdependent behavioral characteristics of a sturgeon species are essentially useful for hatchery managers not only to validate the physiological and developmental fitness of artificially propagated prelarvae but also to develop an appropriate illumination regime for effective prelarval nursery (Gisbert et al., 1999a; Gisbert and Solovyev, 2018). Different light illumination regimes during prelarval or larval stages may give rise to significantly variable outcomes with regard to viability, growth and/or stress response of sturgeons (Ruchin, 2008).

Siberian sturgeon (Acipenser baerii) is a commonly aquacultured species worldwide. The introduction of Siberian sturgeon to the Korean aquaculture area dates back to the late 1990s, and from then on, there have been various researches including artificial seedling propagation, in vitro cell culture and innate immunity (Park et al., 2013a; Ryu et al., 2018; Kim et al., 2019c). Siberian sturgeon is known to exhibit a strong positive phototaxis during its early prelarval life (Gisbert and Ruban, 2003). Ontogenetic development and phototaxis of this sturgeon species have been described previously (Gisbert et al., 1999a; Gisbert and Solovyev, 2018). However, most previous studies have been based on observation data undertaken only under daylight conditions (Gisbert et al., 1999a; Gisbert and Ruban, 2003; Gisbert and Solovyev, 2018). On the contrary, quantitative approaches to investigate behavioral modifications of Siberian sturgeon prelarvae in response to different light intensities have not been extensively studied. Although previous studies have generally remarked that characteristic swimming behaviors observed in daytime conditions would disappear at night (Gisbert and Ruban, 2003; Gisbert and Solovyev, 2018), quantitative data have been unavailable yet. In hatcheries, sturgeon prelarvae are generally expected to experience substantial changes of light intensities according to the diel photoperiodicity, suggesting that the practical guide for prelarval nursery should essentially consider the photobehavior of prelarvae. Furthermore, the comprehensive guideline of behavioral ontogeny may also be a fundamental basis for various research investigations with prelarvae such as ontogenetic gene expression assay and examination of physiological alterations, because all those investigations need the information on the standard behavioral phenotype of experimental specimens used (Song et al., 2016; Kim and Nam, 2018; Kim et al., 2019c). However, despite its importance, quantitative assay of behavioral characteristics of Siberian sturgeon prelarvae in a diel photoperiodicity has been very insufficiently addressed insofar. Accordingly, the objective of this study is to examine behavioral responses of Siberian sturgeon prelarvae to different light illumination intensities (daylight, dimlight and dark) applied in a diel light cycle, and also to scrutinize daily changes of their phototactic degrees using a spot light illumination in dark conditions.

\section{MATERIALS AND METHODS}

\section{Preparation of Siberian sturgeon prelarval samples}

Mature Siberian sturgeon brood fish (11-year-old; average body weight $=21.5 \pm 4.3 \mathrm{~kg} ; \mathrm{n}=2$ each for female and male) were selected based on the visual identification of gametes obtained through the external gonad biopsy (Chapman and Park, 2005). They were given intramuscular injections of luteinizing hormone-releasing hormone analog (LHRH-a; Sigma-Aldrich, St. Louis, MO, USA) at concentrations of $80 \mu \mathrm{g} / \mathrm{kg}$ (for females) and $20 \mu \mathrm{g} / \mathrm{kg}$ (for males). Gametes were collected from each individual according to the procedure described by Park et al. (2013a). Artificial insemination was carried out with wet method at 
$17^{\circ} \mathrm{C}$, and fertilized eggs were treated with anti-adhesive agent (Fuller's earth; Sigma-Aldrich) before being placed in McDonald hatching jars (Park et al., 2013a). The egg incubation temperature was gradually increased to $20^{\circ} \mathrm{C}$ $\left(1^{\circ} \mathrm{C} / \mathrm{h}\right)$, and kept constant at $20 \pm 0.5^{\circ} \mathrm{C}$ until hatching and afterwards. For each cross (two $\times$ two cross), fertilization rate, hatching success and incidence of abnormalities in hatched prelarvae were estimated in triplicates with at least 120 embryos or hatchlings according to the procedures described previously (Park et al., 2013b). Upon mass hatching, similar numbers of newly hatched prelarvae were collected from each mating pair (approximately 10,000 prelarvae per mating pair) and were pooled into a group. Under the present conditions, fertilization rates (96.5\%-98.4\% depending on mating pair), hatching success $(78.5 \%-80.3 \%)$ and incidence of abnormalities (4.1\%$5.2 \%)$ were highly comparable to those reported in previous studies (Park et al., 2013a, 2013b). Overall mortality throughout the prelarval period (Day 0-Day 9) was less than $2 \%$ in all replicate tanks. Described experiment was approved by the Animal Care and Use Committee of $\mathrm{Pu}$ kyong National University (Approval number: 201818). All experimental procedures were performed in accordance with National Act on Laboratory Animals.

\section{Experimental photoperiodicity}

A diel photoperiodic cycle was set in the following order: daylight (L) phase for $16 \mathrm{~h}$, dimlight (DL) phase for $4 \mathrm{~h}$ and darkness (D) for $4 \mathrm{~h}$. Illumination intensities measured above tanks for L, DL and D phases were $500 \mathrm{~lx}, 5 \mathrm{~lx}$ and less than $0.5 \mathrm{~lx}(<0.5 \mathrm{~lx})$, respectively. Light conditions applied in this study are broadly equivalent to general conditions used in domestic sturgeon farms. Our observation regimes for behavioral assessments and phototaxis tests are indicated in Fig. 1. All the windows of the rooms used in this study were blinder-covered to block the interfering effects of unintended external lights. Light sources were white light-emitting diode (LED) room light lamps enclosed with translucent plastic covers. Observations of prelarvae under dark conditions were aided by using an infrared (IR) light illuminator (850 nm) and/or an IR-night vision camera. Behavioral criteria and morphological differentiations of the Siberian sturgeon prelarvae were adopted from the previous descriptions under daylight conditions including swimming-up/drifting, rheotaxis, and schooling (Park et al., 2013a; Gisbert and Solovyev, 2018).

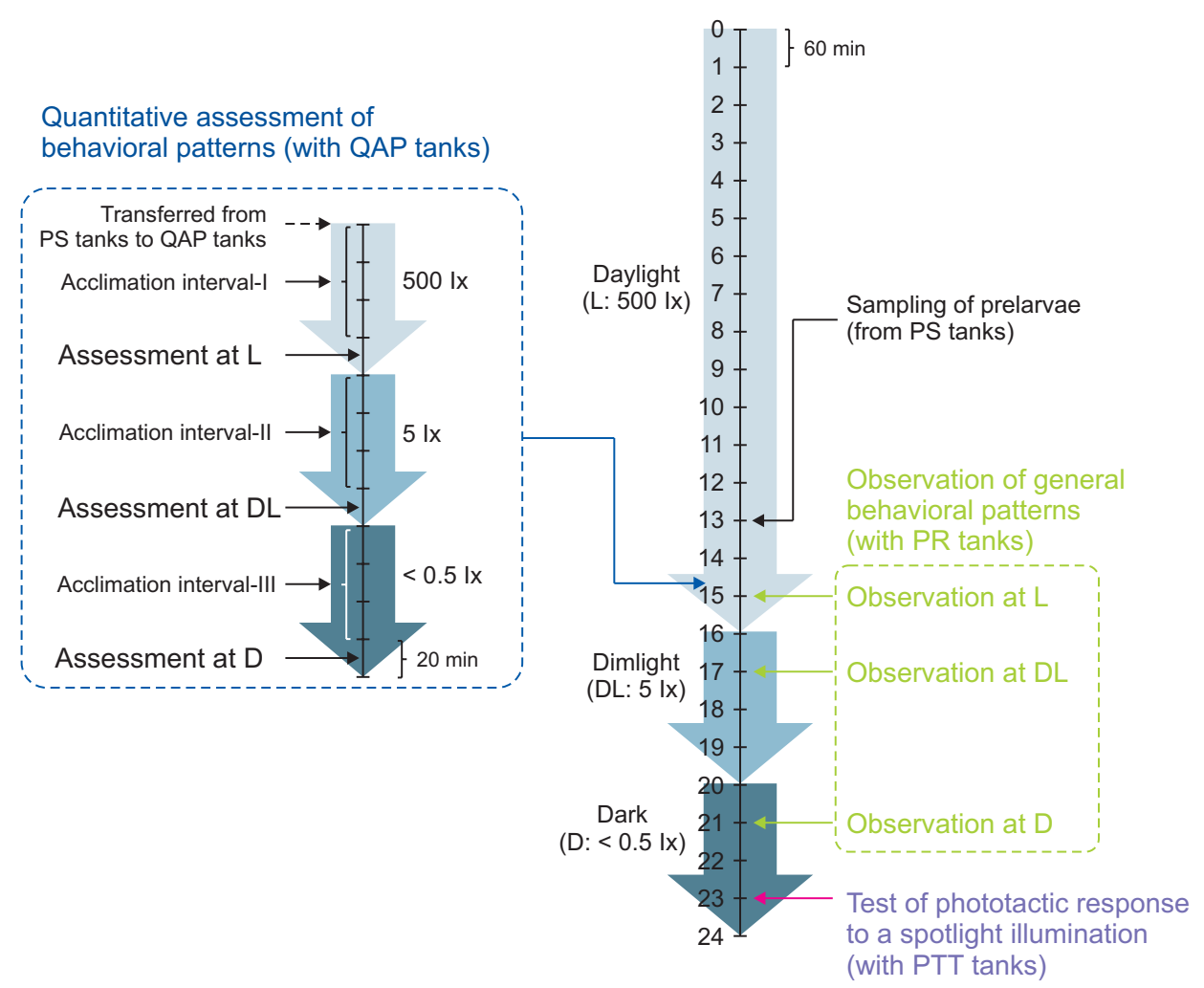

Fig. 1. Observational regimes for behavioral patterns of $A$. baerii prelarvae in response to different light illumination intensities $(<0.5,5$ and $500 \mathrm{~lx}$ ) in a diel photoperiodicity. Abbreviations and purposes of experimental tanks used in this study are PR (prelarval rearing for general behavioral observation), PS (prelarval sampling for validating normal development and prelarval growth), QAP (quantitative assessment of prelarval behavior), and PTT (phototaxis test in response to spot illumination at darkness) tanks. 


\section{Observations of general behavioral patterns in response to a diel light cycle}

In order to examine general patterns of ontogenetic behaviors of Siberian sturgeon prelarvae in response to the diel photoperiodicity set of this study, four rearing tanks leach $2 \mathrm{~m}(\mathrm{~W}) \times 1.2 \mathrm{~m}(\mathrm{D}) \times 0.25 \mathrm{~m}(\mathrm{H})$; termed 'prelarval rearing (PR)' tank in this study] were prepared. Each PR tank was equipped with independent 200-L capacity of external filer unit (custom-designed) to recirculate the water. Tank and filter were filled $1-\mu \mathrm{m}$-filtered ground water to adjust 'in-tank' water depth to be $15 \mathrm{~cm}$ for each tank. Colors of tank bottom and walls were all white. Water current in a radial shape was prepared in each PR tank to induce the rheotacism of prelarvae. The water flow rate was $8 \mathrm{~L} / \mathrm{min}$. Temperature and dissolved oxygen throughout the prelarval period were adjusted to be $20.0 \pm 0.5^{\circ} \mathrm{C}$ and $7.6 \pm 0.5 \mathrm{mg} / \mathrm{L}$, respectively. The water $\mathrm{pH}$ ranged from 7.2 to 7.4 . Randomly chosen 2,400 prelarvae were assigned to one of four replicate PR tanks, and daily changes or transitions of ontogenetic behaviors were observed during the period from Day 0 to Day 9. Dead individuals were immediately removed and recorded. Daily water exchange rate was $10 \%$. In every day, periodical observations of general behavioral patterns were made with PR tanks at $1 \mathrm{~h}$ before the end of the $\mathrm{L}$ phase and $1 \mathrm{~h}$ each after the beginning of the DL and D phases, respectively (Fig. 1).

\section{Quantitative assessments of behavioral patterns}

Because the accurate counting of prelarvae belonging to a given behavioral type was not feasible in PR tanks, quantitative observations were made with small-sized observation tanks containing fewer prelarvae. Six rectangular tanks [35 cm (D) $\times 55 \mathrm{~cm}(\mathrm{~W}) \times 25 \mathrm{~cm}(\mathrm{H})$ each; termed 'quantitative assessment of prelarval behavior (QAP)' tank] as well as two additional tanks [2,400 prelarvae per tank; termed 'prelarval sampling (PS)' tank] of which sizes and other conditions were identical to those of PR tanks above were prepared. All water parameters of QAP tanks were prepared to be as close as possible to those of the PR and PS tanks. At $1 \mathrm{~h}$ and 20 min before the end of the L phase, twenty prelarvae randomly chosen from each PS tank was transferred to one of three QAP tanks (i.e., six QAP replicate tanks in a total) under $\mathrm{L}$ conditions. One hour after the acclimation interval in QAP tanks, relative proportions of prelarvae representing a particular type of behavior criterion were assessed for $20 \mathrm{~min}$. After ob- servations under L conditions, quantitative observations were made similarly under DL and D conditions, with the 1-h acclimation interval between different light intensity stages (Fig. 1). After observations were finished in each day, prelarvae were returned to each PS tank.

From each PS tank, 12 randomly chosen individuals were sampled every day (Day 0 to Day 9) in order to confirm the normal morphological differentiation and growth during the prelarval stage. Total length of each sampled individual was measured to the nearest $0.1 \mathrm{~mm}$, and the 10 individuals ranked at the middle range of total length were subjected to the microscopic examination of morphological development for confirming the normal ontogenetic development.

\section{Navigational response to a spotlight illumination under dark conditions}

Phototactic test was undertaken at $1 \mathrm{~h}$ before the end of the D stage. Three test tanks for examining the phototactic response to a spotlight [termed "phototaxis test (PTT)' tanks] were prepared, in which each tank consisted of 1,200 prelarvae. Except the prelarval number, all other conditions of PTT tanks were identical to those of PR tanks above. A square area $(50 \mathrm{~cm} \times 50 \mathrm{~cm})$ on the bottom of one corner of the PTT tank was line-marked with waterproof ink. Polypropylene plastic-framed 500- $\mu \mathrm{m}$ meshed screens were placed on marked lines to prevent prelarvae from entering the area before spotlight illumination. A white LED flashlight was placed $15 \mathrm{~cm}$ above the water surface in order to provide a focused spotlight in the center of the square area. In order to prevent the illumination condition of each PTT tank from being affected by light sources from neighboring tanks, black partitions were installed between all the replicate PTT tanks. When the LED flashlight was turned on, the light intensity at the water surface of the LED light-focused spot (i.e., the center of the square area) was 8,900-9,000 lx and the light intensity at the edge of the square area was 80-90 lx. On the other hand, the light intensity at corner diagonally opposite from the light source was 10-11 lx. Immediately after the LED flashlight was turned on, the screen was removed in order to allow prelarvae to gather the spotlighted area. After 120-sec spotlight illumination, the PP screens were quickly re-installed on the marked lines in order to hold prelarvae in the spotlight-illuminated area (i.e., $50 \mathrm{~cm} \times$ $50 \mathrm{~cm}$ area). In each PTT tank, the number of prelarvae 
inside the screened sector was counted, or, alternatively, calculated by counting the prelarvae outside the screened area. Proportion (\%) of prelarvae inside the square area out of total number of prelarvae in each PTT tank was addressed as a relative phototactic level (RPL). Three replicate PTT tanks were prepared in order to estimate mean RPLs on each day. Phototaxis tests under dark conditions were made during the period from Day 0 to Day 9.

\section{Statistics}

Means for fertilization, hatching, abnormality, prelarval viability, proportion of behavioral patterns and phototactic levels were assessed by one-way ANOVA test followed by Duncan's multiple range tests. Differences were considered to be significant when $p<0.05$.

\section{RESULTS AND DISCUSSION}

\section{Ontogenetic behavioral responses to different illumination intensities in a diel photoperiodicity}

Behavioral patterns under daylight conditions were broadly accordant with those previously described in this sturgeon species, although onsets and transitions of behaviors were not exactly identical with those of previous reports, which might be in relation with the difference in temperature $\left(20^{\circ} \mathrm{C}\right.$ in this study vs. $16-18^{\circ} \mathrm{C}$ in previous studies) (Gisbert and Williot, 1997; Gisbert, 1999; Gisbert and Ruban, 2003). Prelarval growth pattern was also in agreement with our previous study (Park et al., 2013b). This study revisited their behavioral modifications between L, DL and D conditions in a comparative perspective. Overall behavioral patterns in response to the diel photoperiodicity during Day 0-Day 9 are summarized in Table 1, and more specifically described as follow.

\section{Day 0-Day 2}

In the earliest prelarval interval (Day 0-Day 2), most hatched prelarvae of Siberian sturgeon (Day 0) displayed a typical 'swimming-up and drifting behavior' under daylight conditions (L), characterized by the active swimming-up toward the water surface and passive sinking down and resting on the bottom of the tank. With the progress of developmental time, the drifting and resting behaviors were reduced, and instead, the time of active swimming in the water upper water column was progressively increased (Day 1-Day 2). This innate behavior (i.e., swimming-up/drifting) was significantly influenced by environmental light intensities, in which the reduced light intensity would weaken and even diminish this behavior and make prelarvae become active benthic swimmers (Fig. 2). From the quantitative assessment with QAP tanks, most prelarvae were engaged in the 'swimming-up/drifting' behavior (over 95\%) under L conditions during Day 0 -Day 1 , and then partly replaced by the behavior of pelagic swimming in the upper water column (35\%) on Day 2. Conversely in DL phase, Siberian surgeon prelarvae continued to show similar behavioral patterns, however, their swimming-up behavior seemed to be slightly weakened, and relatively larger proportions of benthic prelarvae were observed, compared to under L conditions (0-5\% vs. 7-20\% of benthic prelarvae under L and DL conditions, respectively). Meanwhile, during the $\mathrm{D}$ phase, behavior of prelarvae was fundamentally changed where most prelarvae stopped the swimming-up behavior and began to benthic movement across the bottom of the tank.

The 'swimming-up/drifting' behavior of sturgeon hatchlings and prelarvae has been known as an evolutionary acquired, strategic means for their adaptations to natural habitats, which is closely related with their dispersal and migration styles to the downstream water (Kynard et al., 2002, 2003, 2010; Chebanov and Galich, 2011). From a hatchery viewpoint, this characteristic behavior has been considered as the first visual criterion to assess the fitness of propagated prelarvae under daylight conditions. Our findings on the light intensity-dependent, behavioral modifications during the period from Day 0-Day 2 suggest that Siberian sturgeon prelarvae should execute the swimming-up movement based on their positive phototactic properties of approaching light signals from the water surface even under fairly low illumination intensity conditions (5 lx). Also, our observational data suggest that a fundamental change from swimming-up behavior to benthic movement of the Siberian sturgeon prelarvae may be triggered in the light intensity close to darkness in the human eye.

\section{Day 3-Day 4}

From Day 3 to Day 4, a behavioral transition from the pelagic swimming in the upper water column (or at the water surface) to benthic movement began under L conditions. Based on the assessment with QAP tanks, the major behavioral pattern under L conditions was still the swim- 


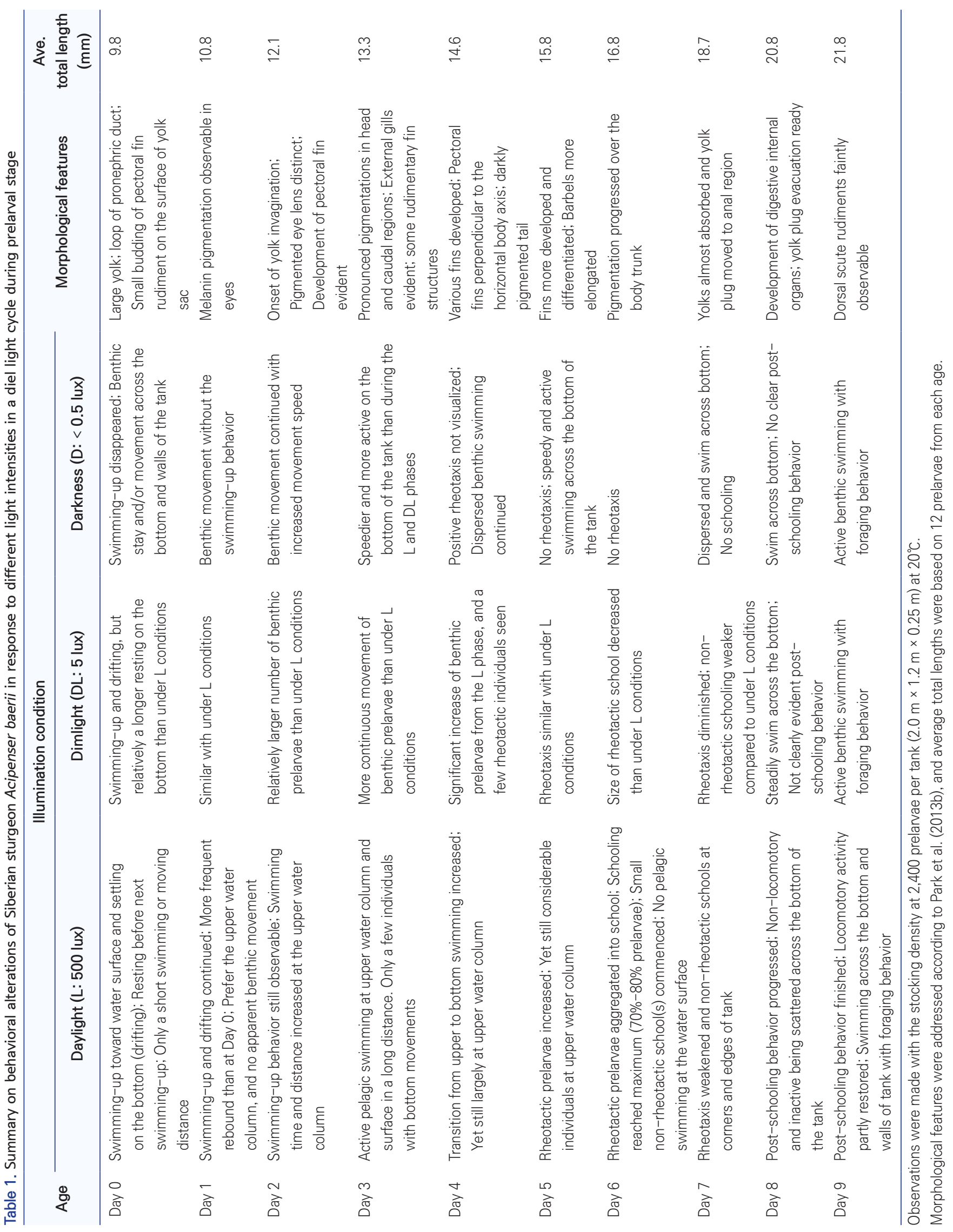




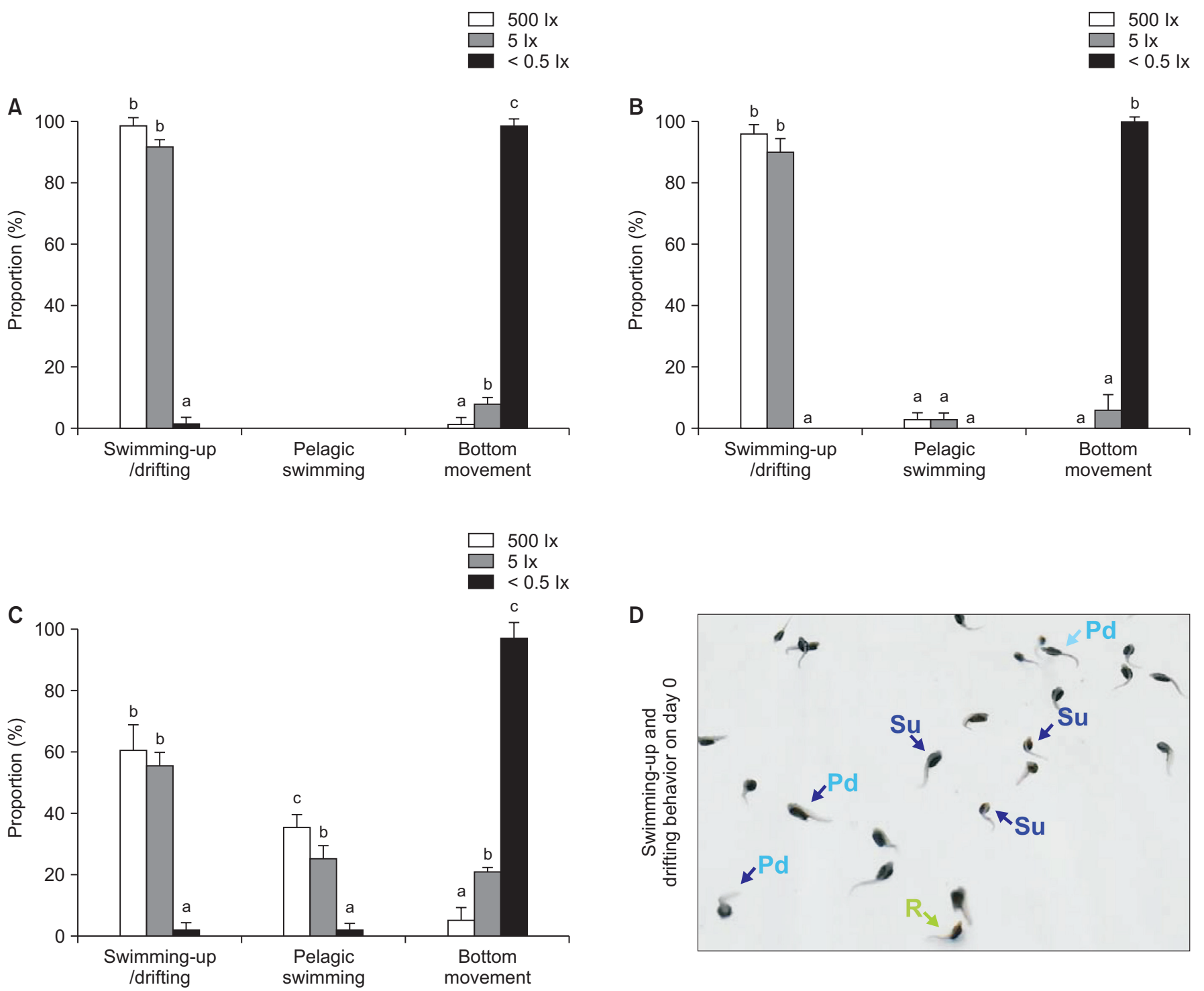

Fig. 2. Behavioral modifications of $A$. baerii prelarvae in response to different light intensities in a diel photoperiodicity on Day 0 (A), Day (B) and Day $3(\mathrm{C})$. A photograph showing the typical swimming-up/drifting behaviors of prelarvae on Day 0 is also provided in (D). In the photograph, prelarvae showing a typical active swimming-up at water surface (indicated by Su), passive drifting or sinking down to bottom $(\mathrm{Pd})$ and the resting on the bottom $(\mathrm{R})$ are noted by arrows. In histograms, means \pm SDs with different letters (a-c) are significantly different based on ANOVA followed by Duncan's multiple range tests at the level of $p<0.05$.

ming in the upper water column ( $75 \%$ to $87 \%$; Day 3 -Day 4). However, compared to the $\mathrm{L}$ phase, more benthic swimmers were observable under DL conditions, which is thought to be because prelarvae are more attracted to a stronger light signal in the L condition than in the DL condition, such that they spend a longer time on the water surface. On Day 4, the proportion of prelarvae engaged in benthic movements under DL conditions (40\%) was fourfold relative to that under L conditions $(10 \%)(p<0.05)$. Conversely, under D conditions, there was no apparent change from the typical behavior of benthic swimming. Taken together, present observations are suggestive of that the vision system may continue to play important roles in the choice of water column (i.e., water surface vs. bottom) by A. baerii prelarvae (Gisbert et al., 1999a; Gisbert and Williot, 2002) (Fig. 3).

\section{Day 5-Day 6}

Assessments with QAP tanks on Day 5 indicated that three behavioral types (i.e., pelagic swimming in the up- 

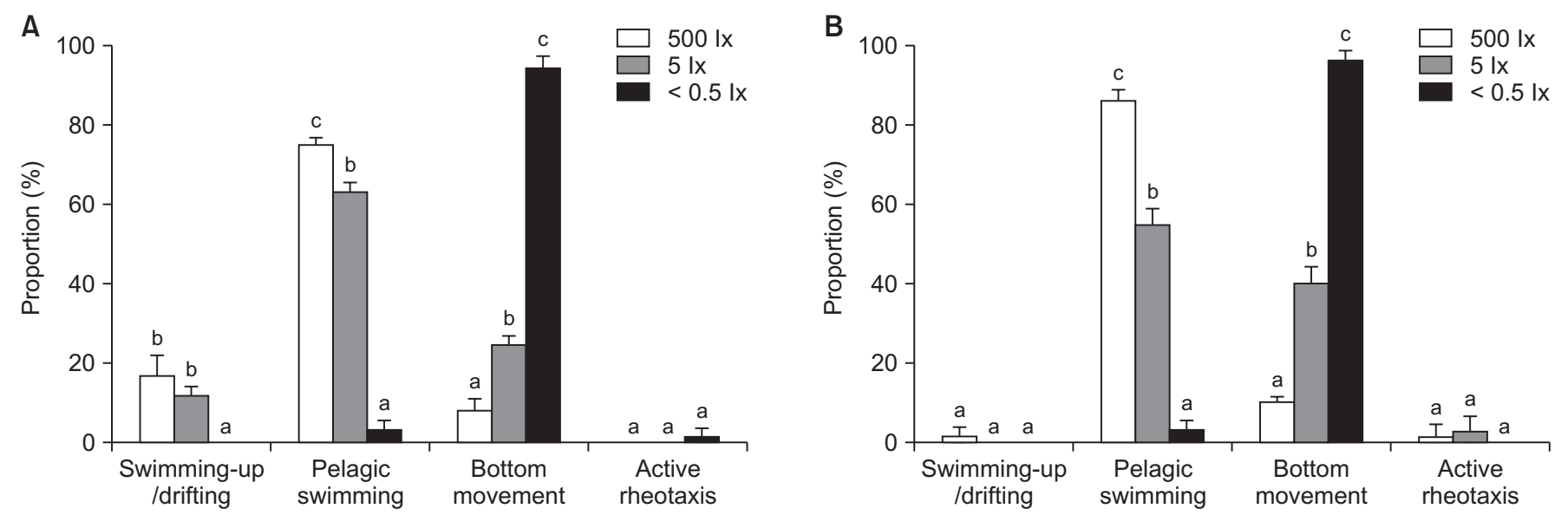

Fig. 3. Behavioral patterns of $A$. baerii prelarvae in response to different light intensities on Day 3 (A) and Day 4 (B). In histograms, means \pm SDs with different letters $(a-c)$ are significantly different based on ANOVA followed by Duncan's multiple range tests at the level of $p<0.05$.
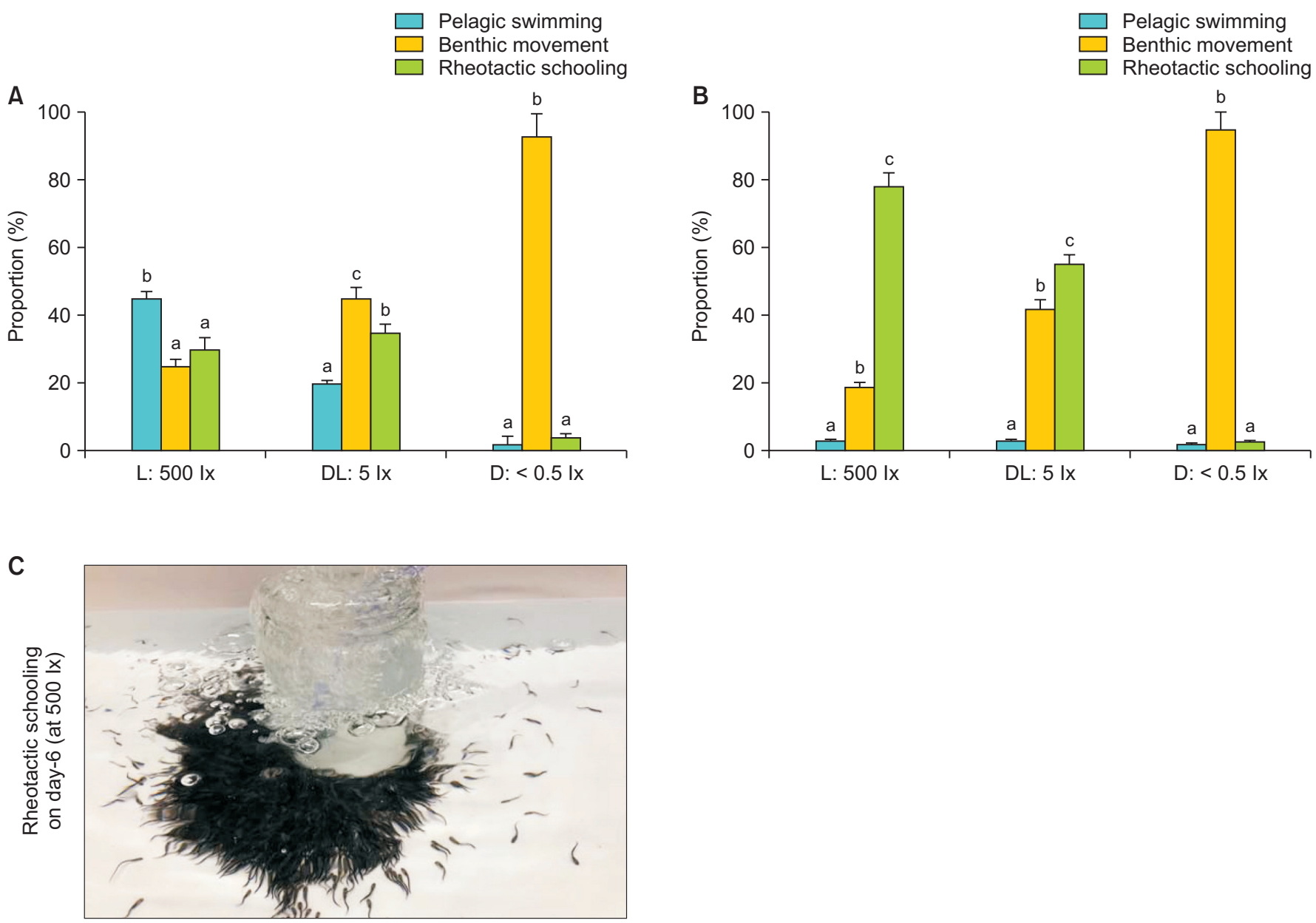

Fig. 4. Effects of light intensities in a diel photoperiodicity on prelarval behavioral patterns (pelagic swimming, benthic movements across the tank and rheotactic schooling) on Day 5 (A) and Day 6 (B). The typical behavioral movement of rheotaxis with the formation of intense school at the site of water current on Day 6 is also shown in (C). In histograms, means \pm SDs with different letters (a-c) are significantly different based on ANOVA followed by Duncan's multiple range tests at the level of $p<0.05$. 
per water column, benthic swimming and rheotactic behavior) would co-exist under both L and DL conditions; however, more prelarvae were engaged in the benthic movement under DL conditions than under L conditions $(p<0.05)$ (Fig. 4). Very few, negligible numbers of rheotactic prelarvae were recorded under D conditions ; however, even if, the rheotactic behavior seemed to be only transient. Appearances of rheotactic schooling behavior (i.e., aggregation of large numbers of prelarvae into the school to face against the water current) observed under $\mathrm{L}$ conditions were no longer observed under D conditions. At L phase of Day 6, the majority of prelarvae joined in the rheotactic schooling. When the schooling reached peak, approximately $70-80 \%$ of prelarvae were engaged in the rheotactic schooling, while remaining prelarvae displayed either the benthic swimming and/or the nonrheotactic schooling at the edge(s) and corner(s) of the tank. However, these large and dense rheotactic schools have not been reliably sustained under DL conditions, in which fewer prelarvae participated in the rheotactic schooling, compared to L conditions. Afterward, upon entering the $\mathrm{D}$ phase, the typical rheotactic schooling behavior was almost disappeared again (Fig. 4).

Ontogenetic acquisition of rheotaxis in sturgeon prelarvae has been reported to be associated with the morphological differentiation of swimming structures and sensory organs (Dettlaff et al., 1993; Boglione et al., 1999; Chai et al., 2006). The active and robust progress of the rheotacism from this interval is in accordance with morphological developments and differentiations of various fins during this period (Park et al., 2013b; Gisbert and Nam, 2018). However, present finding on the reduction and disappearance of the rheotactic schooling behavior under DL and D conditions suggest that the rheotacism of Siberian sturgeon prelarvae may not be completely independent of light factors, and that light signals are indispensable information source for Siberian sturgeon prelarvae to maintain the rheotaxis.

\section{Day 7-Day 9}

On Day 7, rheotaxis became weakened, while postrheotaxis schooling behavior was more robustly observable at edge sides and/or corner(s) of the tank under L conditions. However, unfortunately, this pattern was not clearly reproducible in QAP tanks, because the number of fish contained in QAP tanks might be too small to form a typical clump of schooling prelarvae. As such, the observational data on Day 7 were only based with PR tanks. The non-rheotactic schooling behavior under L conditions was not equally persistent under DL conditions (i.e., partially weakened), and almost disappeared under D conditions. Basically, schooling behavior is considered as a negative phototactic character to avoid the light (Loew and Sillman, 1998). Disappearance of schooling behavior at night hours has been described previously, although specific light intensities at night hours have not been clarified (Gisbert et al., 1999a; Gisbert and Ruban, 2003). On a basis of light intensities tested in this study, the lower limit of the intensity for Siberian sturgeon prelarvae to decide the maintenance of the active schooling behavior may range between $<0.5-51 x$, although effects of other surrounding factors including the stocking density and the water flow rate should be further tested (Chebanov and Galich, 2011; Zaheh et al., 2013). Previous description that prelarval dispersal and downstream migration of this species in natural habitats would occur primarily at nights (Gisbert and Solovyev, 2018) is also congruent with our finding under D conditions.

From Day 8 to Day 9, prelarvae underwent the progress of post-schooling behavior under L conditions with an initial sign of loosening the school(s). Prelarvae were getting out of the school(s), becoming inactive and motionless, and eventually dispersed over the bottom of the tank without locomotory activity. However, results from this study indicate that the post-schooling behavior of this Acipenser species is also substantially influenced by environmental light signals. Under low illumination intensity conditions (particularly under D conditions), large numbers of prelarvae still showed the locomotory activity (i.e., slow benthic movements) (Fig. 5). Previously, postschooling has been described as an ending behavior of the prelarval stage under daylight conditions, in which prelarvae try to channel energy from active movements to final preparations of their digestive and other related endocrinology systems to enter the larval stage (Gisbert et al., 1999b; Chebanov and Galich, 2011). However, we found that the evacuation of yolk plug actively occurred only after they spent a considerable period of postschooling behavior, recommending that the visual criterion to define prelarval stages should be revised in detail with the inclusion of this post-schooling period. Also, our finding suggests that light intensities should be carefully 
considered for hatchery practices to identify more precisely the ending of prelarval stage, which is important for deciding the best timing of the first exogenous feeding (Gisbert et al., 1997; Gisbert, 1999). After the evacuation of yolk plug, prelarvae recovered the locomotory activity and showed the foraging behavior, indicating the transition from endogenous to exogenous nutritional sources (Day 9).

\section{Phototactic response to a spotlight under dark conditions}

Siberian sturgeon prelarvae were able to show a clear positive phototaxis as early as upon hatching (Day 0), in which more than $70 \%$ prelarvae approached the spotlight and gathered inside the spotlight-illuminated area [relative phototactic level $(\mathrm{RPL})=71.6 \pm 2.8 \%]$. The RPL elevated on Day $1(86.4 \pm 1.5 \%)$ and reached peak on Day $2(98.8$ $\pm 0.8 \%)(p<0.05)$. This highest level continued during the period from Day $3(98.4 \pm 0.6 \%)$ and Day 4 (97.8 $\pm 1.1 \%)$. During this period, prelarvae strongly pursued light signals and densely gathered at the water surface below the spotlight source. Further, prelarvae were able to quickly decide their navigation to follow the moving light source. On Day 5, a slight, but statistically significant, reduction of RPL was observed $(94.9 \pm 1.6 \% ; p<0.05)$, and the RPL was further decreased on Day $6(86.0 \pm 3.5 \%)(p<$ 0.05). A marked reduction in RPL was detected on Day 7 $(45.9 \pm 2.5 \%)(p<0.05)$, and the typical scooting behav-

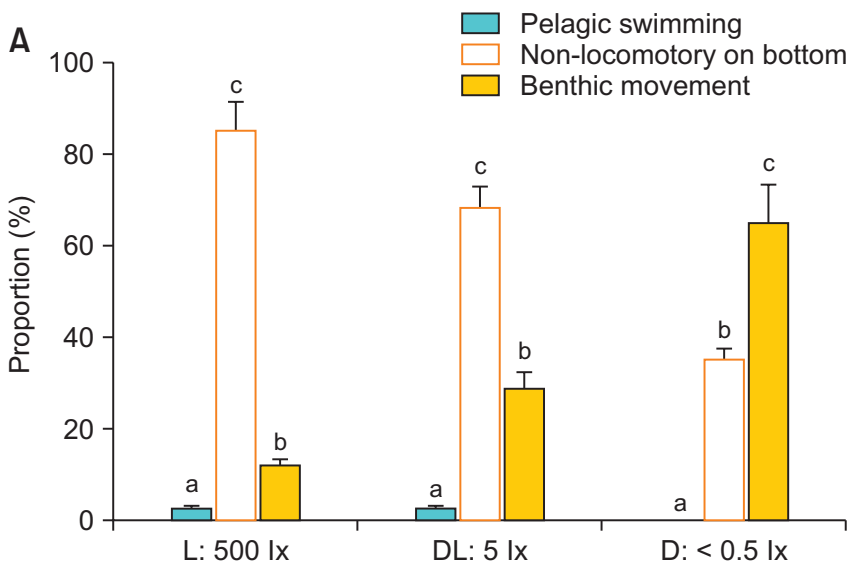

Fig. 5. Percent proportion of $A$. baerii prelarvae engaged in pelagic swimming, post-schooling behavior, or benthic movement across the tank under different light intensity conditions on Day 9. Means \pm SDs with different letters $(a-c)$ are significantly different based on ANOVA followed by Duncan's multiple range tests at the level of $p<0.05$. ior approaching the spotlight source was not observed. A further reduction in RPL was recorded on Day 8 (RPL = $23.9 \pm 1.0 \% ; p<0.05)$, and the strong phototaxis was no longer evident. On the other hand, the RPL was partly rebounded on Day $9(55.9 \pm 1.7 \%)(p<0.05)$ (Fig. 6).

Our findings on the phototactic responses to a spotlight illumination under dark conditions are broadly in agreement with results of previous studies to report the most prominent positive phototaxis of $A$. baerii prelarvae from Day 0 to Day 4 as assessed by the preference testing with differentially illuminated sections (Gisbert et al., 1999a; Gisbert and Ruban, 2003; Nam and Kim, 2019). Early establishment of positive phototaxis is also in agreement with previous observations on the dispersal and migration style of early prelarvae in this sturgeon species (Gisbert and Solovyev, 2018). However, results from this study indicate that Siberian sturgeon prelarvae did not exhibit the full strength of phototaxis on Day 0 and Day 1 when compared to the period from Day 2 to Day 4. This may be related to the incomplete development of the vision system and swimming structure, because wellpigmented eyes and developed fins were not yet evident at these ages (Rodriguez and Gisbert, 2002; Park et al., 2013b). Based on this viewpoint, it is possible that not all the newly hatched larvae were able to reach the spotlight source within the short illumination duration, in spite of their inherent phototactic character. On the other hand,

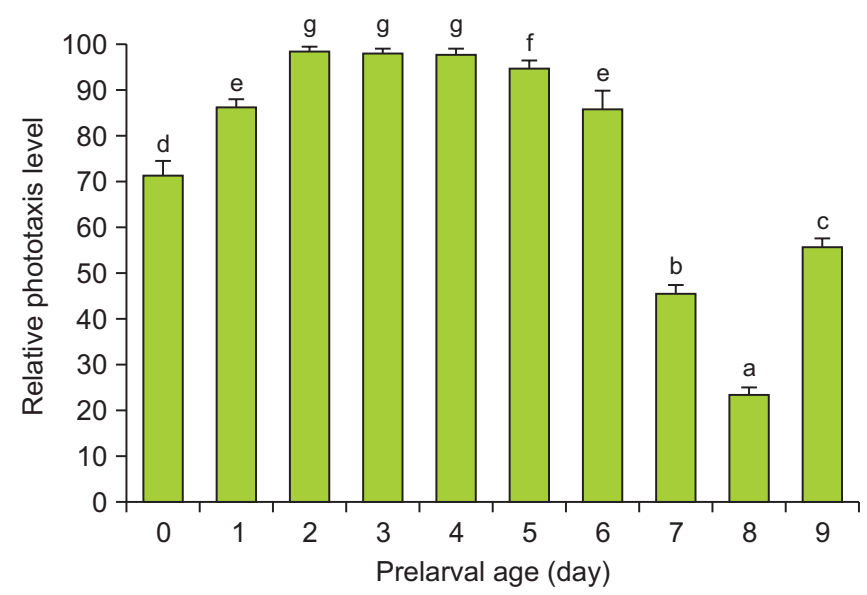

Fig. 6. Temporal changes of relative phototaxis level (RPL) of $A$. baerii during the prelarval stage from Day 0 to Day 9. The RPL values are measured with the spot light illumination under dark conditions, and methods in detail can be referred to Materials and method. Means \pm SDs with different letters (a-g) are significantly different based on ANOVA followed by Duncan's multiple range tests at the level of $p<0.05$. 
our observations on the remarkable reduction of positive phototaxis on Day 7 and Day 8 differ from those in previous experiments undertaken during daylight hours, which reported that the majority of prelarvae (> 90\%) still preferred daylight sections over dimlight-illuminated sections at the same ages (Gisbert et al., 1999a). However, a recent review has remarked that Siberian sturgeon prelarvae may show no phototaxis on Day 7-Day 8 (at $18^{\circ} \mathrm{C}$ ), although specific data was not quantitatively provided (Gisbert and Solovyev, 2018). Such discrepancies may come from differences in test conditions such as intensity of the light source, pre-adaptation under different light intensities, as well as the measuring methodology. For this reason, further comparison should be made in order to pinpoint any potential factor(s) that influence the phototaxis of this species. A rebound of phototactic levels on Day 9 (which coincided with the timing of transition to exogenous feeding) may be related to the strategic establishment of the ability to search for initial food organisms (e.g., small insect larvae and/or zooplankton). Because these food organisms could be also attracted by environmental light during dark hours in nature, the transient acquisition of positive phototaxis in Siberian sturgeon larvae at this stage could increase the chance of successfully acquiring food organisms in their habitats under dark conditions. Previously reports have remarked that fish individuals that do not exhibit a normal light response pattern at a given prelarval interval may often have physiological or developmental deformities (Karlsen et al., 1995; Gisbert and Solovyev, 2018). Hence, data on navigational responses to a spotlight in the present study could be used as a practical means to detect abnormal prelarvae under dark conditions (Gisbert et al., 1999a).

Taken together, the ontogenetic behaviors of Siberian sturgeon prelarvae were substantially affected by light intensities $(0.5 \mathrm{~lx}$ to $500 \mathrm{~lx})$ in the diel photoperiodicity set. Our data on both qualitative and quantitative changes may suggest that anthropogenic lights nearby natural spawning grounds in the night may give rise to potential alterations or interfering effects on ecological behaviors (e.g., dispersal, migration and avoidance of predator) of Siberian sturgeon prelarvae. The range of light intensities addressed in this study is broadly equivalent to that generally applied to domestic Siberian sturgeon hatcheries, and thereby, observational data from this study could also be a useful basis for developing a revised visual guide to bet- ter evaluate the fitness of hatchery-propagated prelarvae. Also, this guide is also valuable to validate the normality of physiology and development of experimental prelarvae in various investigations with research purpose. Quantitative data on phototactic responses to a spotlight at darkness could be of practical value to develop a non-invasive and simple methodology to assess the quality state and normality of the prelarvae under dark conditions. Further investigations are needed to examine effects of additional factors (e.g., rearing temperature, stocking density and water flow rate etc.) on the range of variations in lightdependent behavioral characteristics of this sturgeon species.

\section{CONFLICTS OF INTEREST}

No potential conflict of interest relevant to this article was reported.

\section{ACKNOWLEDGEMENTS}

This research was supported by a Research Grant of Pukyong National University (2019).

\section{AUTHOR CONTRIBUTIONS}

EJK carried out analysis of behaviors including image preparations. CP performed the induced breeding of sturgeon and co-evaluated the data. YKN designed this study, carried out data evaluation and drafted the manuscript. All authors read and approved the final manuscript.

\section{AUTHOR'S POSITION AND ORCID NO.}

EJ Kim, Graduate Student, https://orcid.org/0000-0001-8303-1731

C Park, Chief Executive Officer, https://orcid.org/0000-0003-3589-9864

YK Nam, Professor, https://orcid.org/0000-0001-8870-2098

\section{REFERENCES}

Birstein VJ, Hanner R, DeSalle R. 1997. Phylogeny of the Acipenseriformes: cytogenetic and molecular approaches. Environ. Biol. Fishes 48:127-155.

Boglione C, Bronzi P, Cataldi E, Serra S, Gagliardi F, Cataudella 
S. 1999. Aspects of early development in the Adriatic sturgeon Acipenser naccarii. J. Appl. Ichthyol. 15:207-213.

Chai Y, Xie C, Wei Q, Chen X, Liu J. 2006. The ontogeny of the retina of Chinese sturgeon (Acipenser sinensis). J. Appl. Ichthyol. 22:196-201.

Chapman FA and Park C. 2005. Comparison of sutures used for wound closure in sturgeon following a gonad biopsy. North Am. J. Aquac. 67:98-101.

Chebanov MS and Galich EV. 2011. Sturgeon hatchery manual. Food and Agriculture Organization of the United Nations, Ankara, pp. 1-303.

Cho YS, Douglas SE, Gallant JW, Kim KY, Kim DS, Nam YK. 2007. Isolation and characterization of cDNA sequences of L-gulono-gamma-lactone oxidase, a key enzyme for biosynthesis of ascorbic acid, from extant primitive fish groups. Comp. Biochem. Physiol. B Biochem. Mol. Biol. 147:178190.

Dettlaff TA, Ginsburg AS, Schmalhausen OI. 1993. Sturgeon fishes: developmental biology and aquaculture. SpringerVerlag, Berlin, pp. 1-300.

Doukakis P, Pikitch EK, Rothschild A, DeSalle R, Amato G, Kolokotronis SO. 2012. Testing the effectiveness of an international conservation agreement: marketplace forensics and CITES caviar trade regulation. PLoS One 7:e40907.

Gisbert E. 1999. Early development and allometric growth patterns in Siberian sturgeon and their ecological significance. J. Fish Biol. 54:852-862.

Gisbert E, Nam YK. 2018. Early ontogeny in the Siberian sturgeon. In: Williot P, Nonnotte G, Vizziano-Cantonnet D, Chebanov M (Eds.), The Siberian Sturgeon (Acipenser baerii, Brandt, 1869). Volume 1 - Biology, Springer, Cham, pp. 131157.

Gisbert E and Ruban GI. 2003. Ontogenetic behavior of Siberian sturgeon, Acipenser baerii: A synthesis between laboratory tests and field data. Environ. Biol. Fishes 67:311-319.

Gisbert E, Sarasquete MC, Williot P, Castelló-Orvay F. 1999b. Histochemistry of the development of the digestive system of Siberian sturgeon during early ontogeny. J. Fish Biol. 55:596-616.

Gisbert E and Solovyev M. 2018. Behaviour of early life stages in the Siberian sturgeon. In: Williot P, Nonnotte G, VizzianoCantonnet D, Chebanov M (Eds.), The Siberian Sturgeon (Acipenser baerii, Brandt, 1869). Volume 1 - Biology, Springer, Cham, 2018, pp. 159-172.

Gisbert E and Williot P. 1997. Larval behaviour and effect of the timing of initial feeding on growth and survival of Siberian sturgeon (Acipenser baeri) larvae under small scale hatchery production. Aquaculture 156:63-76.

Gisbert E and Williot P. 2002. Advances in the larval rearing of Siberian sturgeon. J. Fish Biol. 60:1071-1092.

Gisbert E, Williot P, Castelló-Orvay F. 1999a. Behavioural modifications in the early life stages of Siberian sturgeon (Acipenser baerii, Brandt). J. Appl. Ichthyol. 15:237-242.

Karlsen Ø, Mangor-Jensen A, Naas K. 1995. A method for determination of halibut larval fitness. ICES Mar. Sci. Symp.
201:198.

Kim CH, Kim EJ, Nam YK. 2019a. Chondrostean sturgeon hepcidin: an evolutionary link between teleost and tetrapod hepcidins. Fish Shellfish Immunol. 88:117-125.

Kim CH, Kim EJ, Nam YK. 2019c. Subfunctionalization and evolution of liver-expressed antimicrobial peptide 2 (LEAP2) isoform genes in Siberian sturgeon (Acipenser baerii), a primitive chondrostean fish species. Fish Shellfish Immunol. 93:161-173.

Kim DS, Nam YK, Noh JK, Park CH, Chapman FA. 2005. Karyotype of North American shortnose sturgeon Acipenser brevirostrum with the highest chromosome number in the Acipenseriformes. Ichthyol. Res. 52:94-97.

Kim EJ and Nam YK. 2018. Anesthetic protocol for microinjection-related handling of Siberian sturgeon (Acipenser baerii; Acipenseriformes) prolarvae. PLoS One 13:e0209928.

Kim EJ, Park C, Nam YK. 2019b. Ontogenetic behavior of farmbred Russian sturgeon (Acipenser gueldenstaedtii) prelarvae in a diel photoperiodic cycle: behavioral modifications in response to light intensity. Fish. Aquat. Sci. 22:4.

Kynard B, Parker E, Kynard B. 2010. Ontogenetic behavior of Kootenai river white Sturgeon, Acipenser transmontanus, with a note on body color: a laboratory study. Environ. Biol. Fishes 88:65-77.

Kynard B, Zhuang P, Zhang L, Zhang T, Zhang Z. 2002. Ontogenetic behavior and migration of Volga river Russian sturgeon, Acipenser gueldenstaedtii, with a note on adaptive significance of body color. Environ. Biol. Fishes 65:411-421.

Kynard B, Zhuang P, Zhang T, Zhang L. 2003. Ontogenetic behavior and migration of Dabry's sturgeon, Acipenser Dabryanus, from the Yangtze river, with notes on body color and development rate. Environ. Biol. Fishes 66:27-36.

Loew ER and Sillman AJ. 1998. An action spectrum for the light-dependent inhibition of swimming behavior in newly hatched white sturgeon, Acipenser transmontanus. Vision Res. 38:111-114.

Mueller KP and Neuhauss SC. 2010. Behavioral neurobiology: how larval fish orient towards the light. Curr. Biol. 20:R159R161.

Nam YK and Kim EJ. 2019. Ontogenetic behavior and phototactic properties of interspecific hybrid prolarvae produced by crossing female Siberian sturgeon Acipenser baerii and male Russian sturgeon Acipenser gueldenstaedtii. Korean J. Fish. Aquat. Sci. 52:274-282.

Park C, Lee SY, Kim DS, Nam YK. 2013a. Embryonic development of Siberian sturgeon Acipenser baerii under hatchery conditions: an image guide with embryological descriptions. Fish. Aquat. Sci. 16:15-23.

Park C, Lee SY, Kim DS, Nam YK. 2013b. Effects of incubation temperature on egg development, hatching and pigment plug evacuation in farmed Siberian sturgeon Acipenser baerii. Fish. Aquat. Sci. 16:25-34.

Richmond AM and Kynard B. 1995. Ontogenetic behavior of shortnose sturgeon, Acipenser brevirostrum. Copeia 1995: 172-182. 
Rodríguez A and Gisbert E. 2002. Eye development and the role of vision during Siberian sturgeon early ontogeny. J. Appl. Ichthyol. 18:280-285.

Ruchin A. 2008. The effects of permanent and variable illumination on the growth, physiological and hematological parameters of the Siberian sturgeon (Acipenser baerii) juveniles. Zool. Zhurnal 87:964-972. Russian.

Ryu JH, Kim MS, Kang JH, Kim DH, Nam YK, Gong SP. 2018. Derivation of the clonal-cell lines from Siberian sturgeon (Acipenser baerii) head-kidney cell lines and its applicability to foreign gene expression and virus culture. J. Fish Biol. 92:1273-1289.
Song W, Jiang K, Zhang F, Lin Y, Ma L. 2016. RNA-sequencing of the sturgeon Acipenser baeri provides insights into expression dynamics of morphogenic differentiation and developmental regulatory genes in early versus late developmental stages. BMC Genom. 17:564.

Webb MA and Doroshov SI. 2011. Importance of environmental endocrinology in fisheries management and aquaculture of sturgeons. Gen. Comp. Endocrinol. 170:313-321.

Zadeh HE, Rafiee G, Eagderi S, Kazemi R, Poorbagher H. 2013. Effects of different photoperiods on the survival and growth of beluga sturgeon (Huso huso) larvae. Int. J. Aquat. Biol. $1: 36-41$. 